\title{
Routine monitoring and assessment of adults living with HIV: results of the British HIV Association (BHIVA) national audit 2015

A. Molloy ${ }^{1 *}$ (D, H. Curtis ${ }^{2}$, F. Burns ${ }^{3}$, A. Freedman ${ }^{4}$ and on behalf of the BHIVA Audit and Standards Sub-Committee

\begin{abstract}
Background: The clinical care of people living with HIV changed fundamentally as a result of the development of effective antiretroviral therapy (ART). HIV infection is now a long-term treatable condition. We report a national audit to assess adherence to British HIV Association guidelines for the routine investigation and monitoring of adult HIV-1-infected individuals.

Methods: All UK sites known as providers of adult HIV outpatient services were invited to complete a case-note review and a brief survey of local clinic practices. Participating sites were asked to randomly select 50-100 adults, who attended for specialist HIV care during 2014 and/or 2015. Each site collected data electronically using a selfaudit spreadsheet tool. This included demographic details (gender, ethnicity, HIV exposure, and age) and whether 22 standardised and pre-defined clinical audited outcomes had been recorded.
\end{abstract}

Results: Data were collected on 8258 adults from 123 sites, representing approximately 10\% of people living with HIV reported in public health surveillance as attending UK HIV services. Sexual health screening was provided within 96.4\% of HIV services, cervical cytology and influenza vaccination within $71.4 \%$ of HIV services. There was wide variation in resistance testing across sites. Only 44.9\% of patients on ART had a documented 10-year CVD risk within the past three years and fracture risk had been assessed within the past three years for only $16.7 \%$ patients aged over 50 years.

Conclusions: There was high participation in the national audit and good practice was identified in some areas. However improvements can be made in monitoring of cardiovascular risk, bone and sexual health.

Keywords: HIV, Diagnostics, Prevention

\section{Background}

In 2014, an estimated 103,700 people were living with HIV (PLWH) in the UK, with 85,489 seen for HIV care during the year $[1,2]$. Improvements in treatments for HIV mean that life expectancy for PLWH has increased dramatically [3]. However with longer life expectancy we are seeing an increasing proportion of people living with co-morbidities including cardiovascular disease, metabolic complications, and malignancy $[4,5]$. As such, to ensure optimal health outcomes for our patient cohort, primary and secondary health promotion and disease

\footnotetext{
*Correspondence: aoifemolloy@nhs.net

${ }^{1}$ Royal Free London NHS Foundation Trust, Pond Street, London NW3 2QG,

United Kingdom

Full list of author information is available at the end of the article
}

prevention extending beyond the virological and immunological control of HIV is required. The British HIV Association (BHIVA) produces guidelines for the management of HIV infection, in the UK. The process used by the BHIVA has been accredited by the National Institute for Health and Clinical Excellence (NICE). Guidelines are drafted by BHIVA Writing Groups and then placed on the BHIVA website for consultation by all interested parties. The BHIVA guidelines for routine investigation and monitoring of adult HIV-1 infected individuals 2011 recognised the paradigm shift in the treatment of HIV infection and provided guidance on the appropriate monitoring and assessment of adults living with HIV [6]. These guidelines present a consensus regarding the standard assessment and investigation of 
HIV infection from the time of diagnosis and describe the appropriate monitoring of HIV-positive individuals both on and off ART.

In 2015, as part of the national audit programme of the British HIV Association (BHIVA), we audited adherence to the BHIVA guideline on monitoring and assessment of HIV infected individuals, and where relevant, immunisation guidelines. This article reports our findings.

\section{Methods}

All UK sites known to BHIVA as providers of adult HIV services were invited to complete a case-note review and a brief survey of local clinic practices. During June to August 2015 participating sites were asked to randomly select from the local list, 50-100 adults (aged 16 or over), who attended for specialist HIV care during 2014 and/or 2015. The selection was random and was not stratified. The number of cases was selected to ensure maximum participation of sites with varying patient populations. Data at each site were collected electronically using a self-audit spreadsheet tool. This included demographic details (gender, ethnicity, HIV exposure, and age) and whether audited outcomes as listed in Table 1 had been recorded. Some of these were modified from outcomes as specified in guidelines for ease of

Table 1 Demographics of sample

\begin{tabular}{|c|c|c|}
\hline All & 8258 & $100.0 \%$ \\
\hline \multicolumn{3}{|l|}{ Sex } \\
\hline Male & 5482 & $66.4 \%$ \\
\hline Female & 2763 & $33.5 \%$ \\
\hline Transgendered/ transgendering & 9 & $0.1 \%$ \\
\hline Not stated & 4 & $0.0 \%$ \\
\hline \multicolumn{3}{|l|}{ Ethnicity } \\
\hline White & 4853 & $58.8 \%$ \\
\hline Black-African & 2592 & $31.4 \%$ \\
\hline Other & 733 & $8.9 \%$ \\
\hline Not recorded/not stated & 80 & $1.0 \%$ \\
\hline \multicolumn{3}{|l|}{ HIV Exposure } \\
\hline Heterosexual & 4320 & $52.3 \%$ \\
\hline MSM & 3550 & $43.0 \%$ \\
\hline IDU & 153 & $1.9 \%$ \\
\hline Other & 99 & $1.2 \%$ \\
\hline Not recorded/not stated & 136 & $1.6 \%$ \\
\hline \multicolumn{3}{|l|}{ Age } \\
\hline $16-29$ & 674 & $8.2 \%$ \\
\hline $30-49$ & 5001 & $60.6 \%$ \\
\hline $50-69$ & 2396 & 29.0 \\
\hline $70+$ & 172 & $2.1 \%$ \\
\hline Not stated & 15 & $0.2 \%$ \\
\hline
\end{tabular}

assessment. To allow for varying appointment dates, monitoring/procedures recommended as "annual" were interpreted as within 425 days ( 14 months). Similarly six monthly was taken to mean within 243 days (8 months), except in the case of HIV viral load testing where the guideline recommendation is to test 3-6 monthly i.e. within six months, rather than at 6 month intervals. As some HIV clinics do not provide influenza vaccination or cervical cytology, information was sought as to whether individuals had been advised to obtain these elsewhere.

This is the first national audit of adherence to the BHIVA monitoring and assessment guidelines. Data were compared to the targets set in the guidelines where applicable. Individual participating sites received their results via the self-audit spreadsheet tool and could compare them with national results which were presented at the BHIVA Autumn conference 2015 [7]. Statistical analysis was performed using Microsoft Excel. Further analyses assessing the effect of adjusting for patient mix on site-level outcomes and summarising outcomes into meaningful groups and presenting results as a visual dashboard have been performed and reported elsewhere [8].

\section{Results}

Case-note review data was provided for 8258 patients from 123 sites, but only 112 services completed the survey of clinic practices. The 8258 individuals included in the case-note review represent approximately $10 \%$ of PLWH reported in public health surveillance as attending UK HIV services [2]. Two thirds were male, just over half (52.3\%) acquired their HIV heterosexually, the majority were of white ethnicity (58.8\%) and $60.6 \%$ were in the 30-49 year age group (see Table 1). 62.0\% (5119/ 8258 ) of patients had been reviewed within three months up to audit data extraction in June-August 2015 and $96.5 \%$ (7971/8258) had been reviewed within 1 year. While it is difficult to perform a direct comparison, the characteristics of our study population are similar to the reported demographic data on people living with HIV in the UK [1].

Table 2 shows the proportion of individuals achieving the audit standards for all audited recommendations.

\section{Baseline resistance testing}

While the majority of audited cases met the national standard for baseline resistance testing (see Table 1), almost 1 in $5(19.2 \%, n=1586 / 8258)$ individuals did not. However baseline resistance testing was recorded as "not possible" for 47.3\% (750/1586); 7.1\% (112/1586) had neither been tested nor had a sample stored; and for $45.4 \%(720 / 1586)$ it was not known whether this had been done or the question was not answered. 
Table 2 Clinical monitoring standard examined and performance

Clinical Monitoring requirement audited
Whether a baseline HIV resistance test had been
done or sample stored for later testing
Whether HIV viral load measured within past 6 month
Whether adherence assessed within past 425 days
Whether all medication recorded within past 425 days
Whether vaccinated/immune to hepatitis A
Whether hepatitis B serology recorded; whether anti-
surface antibody measured within past 425 days for

surface antibody measured within past 425 days for
individuals with serology consistent with vaccination

Whether hepatitis $\mathrm{C}$ antibody status known

Whether CVD risk assessed, within past 3 years if on ART, ever if not on ART

Whether smoking status recorded within past two years; if a smoker, whether offered a cessation service.

Whether blood pressure recorded within past 425 days

Whether glucose measurement recorded within past 425 days

Whether lipid profile recorded within past 425 days

Whether liver function test (LFT) assessed within past 425 days

Whether estimated glomerular filtration rate (eGFR) assessed within past 425 days

Whether urinalysis or urine protein/creatinine (UP/C) checked within past 425 days, or 243 if receiving tenofovir

Whether flu vaccination had been done or record made of advice to obtain this from general practitioner (GP) within past year: as audit was conducted in summer this fully covered the preceding season

Whether sexual health screen offered within past 425 days

Whether syphilis serology had been done within past 243 days

Whether cervical cytology had been done or record made of advice to obtain elsewhere within past 425 days, females only

Whether bone mineral density measured, individuals aged $>70$ and on ART only

$$
\text { Specified target in guidelines }
$$

Patients with a genotypic resistance test performed within 3 months of first diagnosis (or with a stored sample available for later testing) (90\%).

Patients on ART with HIV viral load measured within the last 6 months (80\%).

Adherence documented within the first 3 months of starting ART (90\%) and at least annually thereafter (70\%).

All medication taken by patients on ART should be reviewed annually (100\%).

No target specified but serology recommended followed by vaccination for all non-immune at risk and/or co-infected with hepatitis B or C.

No target specified but surface antigen ( $\mathrm{HBsAg}$ ), anti-core total antibody (anti-HBC) and antisurface antibody (anti-HBs) testing recommended. Vaccination recommended if non-immune. Annual surface antibody titre measurement recommended in vaccine responders.

No target specified but antibody testing recommended, followed by RNA testing if antibody positive. Annual re-testing recommended for antibody negative men who have sex with men (MSM) or injecting drug users (IDU).

10-year cardiovascular disease (CVD) risk calculated within 1 year of first presentation (70\%), and within the last 3 years if taking ART (70\%).

Smoking history documented in the last 2 years $(90 \%)$ and if a smoker offered referral to a cessation service (90\%).

Blood pressure (BP) recorded in the last year (90\%).

No target specified but recommended yearly or 3-6-monthly if on ART.

No target specified but recommended yearly or 6-12-monthly if on ART.

No target specified but recommended yearly or 3-6 monthly if on ART.

No target specified but recommended yearly or 3-6 monthly if on ART.

No target specified but urinalysis and $\mathrm{UP} / \mathrm{C}$ recommended annually, with 3-6-monthly urinalysis if receiving tenofovir.

No target specified in monitoring guidelines but vaccination history recommended as part of regular clinical review. Vaccination guidelines specify: offer annual influenza vaccination to all HIV-infected persons (target 95\%) [14].

No target specified but recommended to offer sexual health screen 12-monthly, or more frequently if identified risks.

No target specified but recommended 3-6-monthly at each routine visit for MSM and 12-monthly for others No target specified but recommended 12-monthly.

$53.2 \%(1471 / 2763)$

Proportion of cases with documentation of meeting audit standard \% (n/N)

80.8\% (6636/8258 tested, 40/8258 sample stored)

$90.1 \%(6660 / 7395)$

$93.4 \%(6908 / 7395)$

$89.0 \%(6584 / 7395)$

$61.2 \%(5053 / 8258)$

$82.1 \%(6781 / 8258)$

$96.6 \%(7979 / 8258)$

$44.9 \%(3318 / 7395)$ on ART $32.3 \%(279 / 863)$

$65.9 \%(5445 / 8258)$ $45.2 \%(862 / 1905)$ offered cessation

$85.5 \%(7058 / 8258)$

$77 \%(6359 / 8258)$

$83.2 \%(6869 / 8258)$

$97 \%(8013 / 8258)$

$95.5 \%(7887 / 8258)$

$73.7 \%(2050 / 2781)$

$74.8 \%$ (4098/5477) receiving tenofovir

$21.1 \%(1744 / 8258)$ vaccination given

36.2\% (2993/8258) advice given

$65.7 \%(5424 / 8258)$

No target specified but recommended in all men aged 70 years and all women aged 65 years. 
Table 2 Clinical monitoring standard examined and performance (Continued)

\begin{tabular}{|c|c|c|}
\hline $\begin{array}{l}\text { Whether fracture risk assessed within past } \\
3 \text { years, individuals aged }>50 \text { only }\end{array}$ & $\begin{array}{l}\text { No target specified but recommended 3-yearly } \\
\text { if aged over } 50 \text { years. }\end{array}$ & $16.7 \%(430 / 2568)$ \\
\hline $\begin{array}{l}\text { Outcome: whether vaccinated against } \\
\text { pneumococcus, CD4 > } 200 \text { only }\end{array}$ & $\begin{array}{l}\text { No target specified in monitoring guidelines but } \\
\text { vaccination history recommended as part of regular } \\
\text { clinical review. Vaccination guidelines recommend } \\
\text { pneumococcus vaccination if CD4 > 200, and } \\
\text { consideration of vaccination at lower CD4. }\end{array}$ & $26.4 \%(2082 / 7877)$ \\
\hline
\end{tabular}

$A R T$ antiretroviral therapy

\section{Antiretroviral treatment}

Of $89.5 \%$ (7395/8258) audited individuals who were on ART, 90.1\% (6660/7395) had a HIV viral load measurement within the past 6 months, exceeding the guideline target of $80 \%$, and $93.4 \%(6908 / 7395)$ had had ART adherence assessed in the last year, which outperformed the target of $70 \%$. However, only $89.0 \%$ (6584/7395) had all other medications recorded within the last year, despite a target of $100 \%$ to avoid drug-drug interactions.

\section{Viral hepatitis}

Among audited individuals, 61.2\% (5053/8258) were hepatitis A vaccinated, immune, or seropositive, $11.9 \%$ $(n=983 / 8258)$ were seronegative, $0.02 \%$ (2/8258) equivocal, and for $26.9 \%(2220 / 8258)$ it was not known whether this had been done or the question was not answered.

In terms of hepatitis $\mathrm{B}, 82.1 \%(6781 / 8258)$ individuals had serology fully reported (hepatitis B surface antigen HBsAg, hepatitis B core antibody (anti-HBc) and hepatitis B surface antibody (anti-HBs)); 0.9\% (72/8258) were HBsAg positive with incomplete antibody status; $10.2 \%$ (841/8258) were HBsAg negative with incomplete antibody status; 6.8\% (564/8258) had unknown HBsAg status or the question was not answered. Among 306 chronic hepatitis B infected (HBsAg+) individuals, 7.8\% (24/306) were apparently unvaccinated and seronegative for hepatitis A. Among 3605 individuals whose status was consistent with vaccination (anti-HBs+, anti-HBc- and HBsAg-), $67.0 \%$ (2416) had had an annual anti-HBs measurement.

Hepatitis $\mathrm{C}$ antibody (anti-HCV) status was negative for $91.3 \%(7539 / 8258)$ individuals, positive for $5.3 \%$ (439/8258), equivocal for $0 \%(1)$, not known or the question was not answered for $3.4 \%(279 / 8258)$. Among the 7539 seronegative individuals, $65.4 \%$ (4928) had had an annual re-test including $74.1 \%(2423 / 3270)$ men who have sex with men (MSM) and 61.8\% (21/34) injecting drug users (IDU), groups in whom this is recommended. Hepatitis C RNA (ribonucleic acid) testing had been done for $91.1 \%(400 / 439)$ anti-HCV positive patients. Data on HCV genotyping were not collected.

\section{Cardiovascular health}

Only 44.9\% (3318/7395) of patients on ART had a documented 10-year CVD risk within the past three years. Of those not on ART, 32.3\% (279/863) had 10-year CVD risk recorded at any time, despite targets of $70 \%$ for both groups. ART status was unclear for 18 patients. Among 1582 patients aged $>50$ years on ART, with no documentation of established CVD, almost half (48.7\%; 770) had CVD risk calculation recorded within the last 3 years.

Smoking status had been documented within the past two years for $65.9 \%(5445 / 8258)$ audited patients, well below the target of $90 \%$. Only $45.2 \%$ (862/1905) of current smokers had been offered a cessation service although this is recommended [9]. Current smokers were not more likely to have CVD risk calculated than ex or never smokers.

\section{Sexual and reproductive health}

An annual sexual health screen was recorded as offered for $65.7 \%(5424 / 8258)$ of all patients, including $72.7 \%(2581 / 3550)$ MSM, and $60.8 \%$ (2627/4320) heterosexuals. Syphilis serology was recorded within the past eight months (243 days) for 63.0\% (5201/ $8258)$ of all patients, $73.4 \%(2604 / 3550)$ MSM, and $55.3 \%(2390 / 4320)$ heterosexuals.

Out of 2763 women, $53.2 \%$ (1471) had annual cervical cytology done and $21.9 \%$ (604) had been advised to attend a GP or sexual health clinic for this. The self-audit spreadsheet tool did not provide an option for women ineligible for cervical cytology. Contraception was reported not relevant for $31.7 \%(877 / 2763)$ of women. It had been discussed for $63.0 \%(1188 / 1886)$ women for whom it was relevant.

\section{Bone health}

Fracture risk had been assessed within the past three years for only $16.7 \%(430 / 2568)$ patients aged over 50 years. Bone mineral density had been measured in $17.4 \%$ (29/167) individuals aged over 70 years and receiving ART. For simplicity, the spreadsheet recorded this from age 70 for both sexes although guidelines recommend it from age 65 in females.

\section{Variation in monitoring between participating sites}

There was wide variation in resistance testing across sites. 27\% (33/123) sites met the target, having a recorded resistance test or stored sample for $>90 \%$ of audited individuals while 27\% (33/123) sites achieved 
this outcome for $<75 \%$ of audited individuals, including $4.9 \%(6 / 123)$ sites who did so for $<60 \%$.

There was variation between sites in the proportion of patients for whom HBsAg status was not known, and for 8 sites this exceeded 20\%. There was wide variation across sites in provision of influenza vaccination with $16 / 123$ sites having administered or advised obtaining influenza vaccination at their general practitioner (GP) or Pharmacy for fewer than $10 \%$ of patients and $17 / 123$ sites for more than $90 \%$ of patients.

There was wide variation in CVD risk calculation between sites; of 122 sites submitting data for patients receiving ART, $21.3 \%$ (26) met the $70 \%$ target for CVD risk recording within three years but 27.9\% (34) achieved this for fewer than $20 \%$ of patients. There was also variation across sites for fracture risk assessment.

\section{Monitoring frequency}

Most survey respondents routinely reviewed patients who were stable on antiretroviral therapy every 6 months, when HIV viral load (VL) was also checked. CD4 counts were less frequently measured (see Table 3 ). Sexual health screening was provided within 96.4\% (108/123) of HIV services, cervical cytology within $71.4 \%(80 / 123)$ of HIV services and influenza vaccination within $71.4 \%(80 / 123)$ of services.

\section{Discussion}

There was high participation in the national audit and the data showed good practice in some areas. Monitoring of ART was performed well; the proportions of individuals with viral load measurement and adherence assessment exceeded the expected standard. CD4 counts were less frequently measured in line with recent evidence on CD4 count monitoring frequency [9]. The proportion of individuals with all medications recorded was high. Sexual health screening was performed well, perhaps due to the availability of this service in the same or adjacent clinics. Overall recording of hepatitis serology was high but with wide variation between sites. There was also wide variation in influenza vaccination, recording of baseline resistance testing, and monitoring of

Table 3 Frequency of routine monitoring for individuals virologically stable ${ }^{a}$ on ART (112 clinical services)

\begin{tabular}{llll}
\hline & $\begin{array}{l}\text { Clinical } \\
\text { review }\end{array}$ & $\begin{array}{l}\text { HIV viral load } \\
\text { measurement }\end{array}$ & $\begin{array}{l}\text { CD4 count } \\
\text { measurement }\end{array}$ \\
\hline 3 monthly & $4(3.6 \%)$ & $6(5.4 \%)$ & $5(4.5 \%)$ \\
4 monthly & $17(15.5 \%)$ & $24(21.4 \%)$ & $16(14.3 \%)$ \\
6 monthly & $74(66.1 \%)$ & $78(69.6 \%)$ & $42(37.5 \%)$ \\
Yearly & $10(8.9 \%)$ & $1(0.9 \%)$ & $45(40.2 \%)$ \\
Other or not answered & $7(6.3 \%)$ & $3(2.7 \%)$ & $4(3.6 \%)$ \\
\hline
\end{tabular}

${ }^{a}$ virologically stable $=$ HIV viral load undetectable on standard assay ( $<50$ copies $/ \mathrm{ml})$
CVD risk factors and bone health. Some sites reported very low rates of baseline resistance testing though it is possible that this may reflect patients diagnosed and commenced on ART before routine resistance testing was available or recommended [10,11].

Cardiovascular disease and smoking are major causes of morbidity and mortality in people living with HIV [4, 12]. However, low recorded rates of monitoring of cardiovascular health were noted and smoking status was not reported for one in seven patients, and less than half of current smokers were offered cessation support. The growing population of older patients living with HIV were also overlooked in terms of bone health, with very low rates of fracture risk assessment and bone mineral density recording. It is not clear if low recorded rates of monitoring of bone density was due to a lack of availability of bone densitometry measurement.

At some sites, a significant proportion of patients had unknown HBsAg status and many patients remain susceptible to hepatitis A, including those with documented chronic hepatitis B infection, for whom hepatitis A could be more serious. There was very low reported coverage of influenza and pneumococcal vaccination, perhaps indicating an issue for patients when responsibility and funding is shared between primary and secondary care.

\section{Limitations}

Participating sites were asked to select individuals randomly from those attending during 2014 and/or 2015, but it is possible that more recent, and perhaps more regular, attenders were over-sampled as suggested by the fact that $62.0 \%$ of individuals had been reviewed within three months and $96.5 \%$ within one year preceding the audit. This may have led to over-estimation of rates of performance and recording of routine interventions.

There was also difficulty in interpreting some targets in the BHIVA guidelines. For example the $90 \%$ target for baseline resistance testing (or sample storage) is applicable for new incident diagnoses but less clear for individuals established in HIV care. As baseline resistance has ongoing clinical relevance, it is important this information remains accessible, including when individuals transfer care between clinics. We considered the target not met (80.8\% individuals having recorded test or sample stored) although only $1.4 \%$ were known to be untested and some of these may have started ART before resistance testing was in use. As for all the monitoring outcomes some findings may reflect incomplete recording or reporting rather than performance.

\section{Conclusion}

Clinical services should review and develop systems to prompt both performance and recording of recommended interventions. Some sites have an 'annual review' system 
or electronic reminders for monitoring and, if adopted more widely, these may improve compliance with the monitoring guidelines. There may be a perception that primary care is responsible for certain monitoring and services such as cardiovascular risk and providing most vaccinations. Hence greater clarity from commissioners about where responsibility lies in these areas, particularly in what the forthcoming tariffs for HIV care cover, will help HIV services focus on areas they are responsible for. Improved communication between HIV and primary care services would streamline and improve care for people living with HIV. Clinical services should also develop improved strategies to care for older patients living with HIV [13].

\begin{abstract}
Abbreviations
anti-HBc: Hepatitis B core antibody; anti-HBs: Hepatitis B surface antibody; anti-HCV: Hepatitis C antibody; ART: Antiretroviral therapy; BHIVA: British HIV Association; BP: Blood pressure; CVD: Cardiovascular disease; eGFR: Estimated glomerular filtration rate; GP: General practitioner; GU: Genitourinary; HBsAg: Hepatitis B surface antigen; HIV: Human immunodeficiency virus; IDU: Injecting drug users; LFT: Liver function tests; MSM: Men who have sex with men; NHS: National health service; PLWH: People living with HIV; RNA: Ribonucleic acid; UK: United Kingdom; UP/C: Urine protein/creatinine
\end{abstract}

\section{Acknowledgements}

Members of the BHIVA Audit and Standards Sub-Committee: A Freedman (chair), B Angus, D Asboe, F Burns, R Byrne, D Chadwick, D Churchill, H Curtis (co-ordinator), V Delpech, K Doerholt, A Molloy, J Musonda, N Naous, O Olarinde, E Ong, S Raffe, C Sabin, A Sullivan. The committee and authors would like to thank all HIV clinical services who submitted audit data, and S Michael, M May and M Gompels of Bristol University for conducting further analyses.

Participating clinical services: Area Infection Unit, Monklands Hospital, Airdrie; Tameside and Glossop Centre for Sexual Health, Ashton Primary Care Centre, Ashton-under-Lyne; Department of GU Medicine, Ysbyty Gwynedd Hospital, Bangor; Department of Integrated Sexual Health and HIV Medicine, Barking Community Hospital, Barking; Clare Simpson Clinic, Barnet General Hospital, Barnet; Department of GU Medicine, Barnsley Hospital NHS Foundation Trust, Barnsley; Department of Sexual Health, Solent NHS Trust, Basingstoke; Department of GU Medicine, Royal United Hospital, Bath; Beckenham Beacon Sexual Health Clinic, Beckenham Hospital, Beckenham; Department of GU Medicine, Bedford Hospital, Bedford; Department of GU Medicine, Royal Hospitals Trust, Belfast; Department of GU Medicine, Birmingham Heartlands Hospital, Birmingham; Department of HIV Medicine, Queen Elizabeth Hospital, Birmingham; Department of GU Medicine, Blackburn Royal Infirmary, Blackburn; The Pannel Suite Clinic, Royal Bournemouth Hospital, Bournemouth; Lawson Unit (HIV) Outpatients, Royal Sussex County Hospital, Brighton; Department of Immunology, Southmead Hospital, Bristol; Department of GU Medicine, West Suffolk Hospital, Bury St Edmunds; Department of GU Medicine, Frimley Park Hospital, Camberley; Infectious Diseases \& HIV/GU Medicine Clinic 1A, Addenbrooke's Hospital NHS Trust, Cambridge; The Gate Clinic, Kent \& Canterbury Hospital, Canterbury; Department of GU Medicine, Cardiff Royal Infirmary, Cardiff; Infectious Diseases Unit, University Hospital of Wales, Cardiff; Department of GU Medicine, St Helier Hospital, Carshalton; Department of GU Medicine, Mid Essex Hospital Services NHS Trust, Chelmsford; The Blanche Heriot Unit, St Peter's Hospital, Chertsey; Department of GU Medicine, Countess of Chester Hospital NHS Trust, Chester; Department of GU Medicine, Chesterfield Royal Hospital NHS Foundation Trust, Chesterfield; Fletcher Unit of Sexual Health, St Richard's Hospital, Chichester; Department of GU Medicine, Essex County Hospital, Colchester; Department of Sexual Health, Leighton Hospital, Crewe; Department of GU Medicine, Croydon University Hospital, Croydon; Department of GU Medicine, Derbyshire Royal Infirmary NHS Trust, Derby; Department of GU Medicine, Dewsbury and District Hospital, Dewsbury; Department of GU Medicine, Downe Hospital, Downpatrick; Department of GU Medicine, Russells Hall Hospital, Dudley; Department of GU Medicine, University Hospital of North Durham, Durham; Department of Sexual Health, Avenue House Sexual Health Clinic, Eastbourne; Department of GU
Medicine, Lothian University Hospitals, Edinburgh; Regional Infectious Diseases Unit, Western General Hospital, Edinburgh; Exeter NHS Walk-in Centre, Exeter; Borders Sexual Health, Galashiels; Trinity Square Health Centre, Gateshead; Department of GU Medicine, Gartnaval General Hospital, Glasgow; Department of GU Medicine, Gloucestershire Royal Hospital, Gloucester; Department of GU Medicine, Orsett Hospital, Grays; Department of GU Medicine, Bure Clinic, James Paget Hospital NHS Trust, Great Yarmouth; Department of Sexual Health, Buryfields Clinic, Guildford; Department of GU Medicine, Northwick Park Hospital, Harrow; Sexual Health Clinic, Station Plaza Health Centre, Hastings; Gaol Street Health Centre, Wye Valley NHS Trust, Hereford; Department of GU Medicine, Wycombe General Hospital, High Wycombe; Department of GU Medicine, Huddersfield Royal Infirmary, Huddersfield; Sexual Health Clinic, Wilberforce Health Centre, Hull; The Oak Tree Centre, Huntingdon; Orwell Clinic, Ipswich; Department of Sexual Health, West Middlesex University Hospital, Isleworth; Department of Infectious Diseases, Worcestershire Acute Hospitals NHS Trust, Kidderminster; Department of GU Medicine, Queen Elizabeth Hospital, King's Lynn; The Wolverton Centre, Kingston Hospital, Kingston upon Thames; Department of Sexual Health, Whytemans Brae Hospital, Kirkcaldy; Leatherhead GUM Clinic, Leatherhead Hospital, Leatherhead; Department of GU Medicine, Leeds General Infirmary, Leeds; Infection and Travel Medicine Unit and GU Medicine, Leeds Teaching Hospitals NHS Trust, Leeds; Tropical and Infectious Disease Unit, Royal Liverpool University Hospital, Liverpool; Department of GU Medicine, Royal Glamorgan Hospital, Llantrisant; 56 Dean Street, Chelsea and Westminster Hospital, London; Caldecot Centre, King's College Hospital, London; Centre for Sexual Health and HIV Research, Mortimer Market Centre, London; Department of GU Medicine, University Hospital Lewisham, London; Department of GU Medicine/HIV, Newham University Hospital, London; Department of HIV Medicine, Royal Free London NHS Foundation Trust, London; Department of Sexual Health, Homerton University Hospital NHS Foundation Trust, London; Harrison Wing, St Thomas' Hospital, London; HIV/GUM Directorate: St Stephen's Centre, Chelsea and Westminster Hospital, London; Jefferiss Wing Centre for Sexual Health, Imperial College Healthcare NHS Trust, London; Patrick Clements Clinic, Central Middlesex Hospital, London; The Ambrose King Centre, The Royal London Hospital, London; Trafalgar Clinic, Queen Elizabeth Hospital, London; West London Centre for Sexual Health, Charing Cross Hospital, London; Department of GU Medicine, Luton \& Dunstable Hospital NHS Trust, Luton; Rubin Clinic, Department of GU Medicine, Maidstone Hospital, Maidstone; Department of GU Medicine, Manchester Royal Infirmary, Manchester; Department of Infectious Diseases, James Cook University Hospital, Middlesbrough; Department of GU Medicine, New Croft Sexual Health Centre, Newcastle upon Tyne; Department of Infection \& Tropical Medicine, Royal Victoria Infirmary, Newcastle upon Tyne; Department of GU Medicine, Cordell Centre, Royal Gwent Hospital, Newport; Sexual Health Service, St Mary's Hospital, Newport; Department of Sexual Health, Northampton General Hospital, Northampton; Department of GU Medicine, Grove Clinic, Norfolk \& Norwich University Hospital, Norwich; Department of GU Medicine and HIV, Nottingham City Hospital, Nottingham; Department of GU Medicine, George Eliot Hospital NHS Trust, Nuneaton; Oldham Sexual Health Service, Royal Oldham Hospital, Oldham; Department of GU Medicine, Oxford Radcliffe NHS Trust, Oxford; Department of Infectious Diseases, Oxford Radcliffe NHS Trust, Oxford; iCaSH Peterborough, Peterborough and Stamford NHS Foundation Trust, Peterborough; Department of GU Medicine, St Mary's Hospital, Portsmouth; Florey Unit, Royal Berkshire Hospital, Reading; Department of GU Medicine, Doncaster \& Bassetlaw Hospitals NHS Foundation Trust, Retford; Department of Sexual Health, Glan Clwyd District General Hospital NHS Trust, Rhyl; Department of GU Medicine, Rotherham NHS Foundation Trust, Rotherham; Department of GU Medicine, Hospital of St Cross, Rugby; Sexual Health Department, Salisbury District Hospital, Salisbury; Department of GU Medicine, Royal Hallamshire Hospital, Sheffield; One to One Centre, Shiremoor; HIV/GUM Directorate, Upton Hospital, Slough; Department of GU Medicine, Ealing Hospital, Southall; Department of GU Medicine, Royal South Hants Hospital, Southampton; Department of GU Medicine, Southport \& Ormskirk NHS Trust, Southport; Department of GU Medicine, St Helen's and Knowsley Hospital, St Helens; Department of Microbiology and GU Medicine, Jersey General Hospital, St Helier; Department of GU Medicine, Sunderland Royal Hospital, Sunderland; Department of GU Medicine, Sherwood Forest Hospitals NHS Foundation Trust, Sutton-in-Ashfield; Department of GU Medicine, Singleton Hospital, Swansea; Department of GU Medicine, Musgrove Park Hospital, Taunton; Department of GU Medicine, Royal Cornwall Hospital, Truro; Department of GU Medicine, The Hillingdon Hospital, Uxbridge; Josephine Butler Centre, King Street Health Centre, Wakefield; Department of GU Medicine, South Warwickshire General Hospitals NHS Trust, Warwick; Department of GU Medicine, Weymouth Community Hospital, 
Weymouth; Department of GU Medicine, Arrowe Park Hospital, Wirral; Sexual Health Services, Worthing; Department of GU Medicine, York Hospitals NHS Trust, York.

\section{Funding}

The authors received no financial support.

\section{Availability of data and materials}

The datasets generated and analysed for this study are not publicly available due to the risk of breaching participant confidentiality via deductive disclosure, particularly as some groups within our dataset are very small.

\section{Authors' contributions}

The project was conceived by AF, HC and FB in collaboration with the members of the BHIVA Audit and Standards Sub-committee. The project was co-ordinated by HC. Data analysis and interpretation was performed by HC and AM. AM drafted the manuscript. All authors read and approved the final manuscript.

\section{Ethics approval and consent to participate}

Ethical approval and informed consent were not required as this study was a clinical audit based on routinely collected data.

\section{Consent for publication}

Not applicable.

\section{Competing interests}

The authors declare that they have no competing interests.

\section{Publisher's Note}

Springer Nature remains neutral with regard to jurisdictional claims in published maps and institutional affiliations.

\section{Author details}

${ }^{1}$ Royal Free London NHS Foundation Trust, Pond Street, London NW3 2QG, United Kingdom. ${ }^{2}$ British HIV Association, c/o Mediscript Ltd, 1 Mountview Court, 310 Friern Barnet Lane, London N20 0LD, United Kingdom. ${ }^{3}$ Royal Free London NHS Foundation Trust AND Research Department of Infection \& Population Health, University College London, Pond Street, London NW3 2QG, United Kingdom. ${ }^{4}$ British HIV Association AND Clinical Reader in Infectious Diseases and Honorary consultant Physician in General Medicine \& Infectious Diseases at University Hospital of Wales, Heath Park, Cardiff CF14 4XW, United Kingdom.

Received: 16 May 2017 Accepted: 28 August 2017

Published online: 13 September 2017

\section{References}

1. Public Health England. HIV in the UK - situation report 2015 incidence, prevalence and prevention. Prot Improv Nations Heal. 2015;1(1):1-20. https://www.gov.uk/government/organisations/public-health-england

2. Public Health England, 2015. HIV New Diagnoses, Treatment and Care in the UK 2015 report: data to end 2014. https://www.gov.uk/government/ uploads/system/uploads/attachment_data/file/469405/HIV_new_diagnoses treatment_and care 2015 report20102015.pdf.

3. May MT, Gompels M, Delpech V, et al. Impact on life expectancy of HIV-1 positive individuals of CD4+ cell count and viral load response to antiretroviral therapy. AIDS. 2014;28(8):1193-202. doi:10.1097/QAD.0000000000000243.

4. Smith CJ, Ryom L, Weber $R$, et al. Trends in underlying causes of death in people with HIV from 1999 to 2011 (D:a:D): a multicohort collaboration. Lancet (London, England). 2014;384(9939):241-8. doi:10.1016/S0140-6736(14)60604-8.

5. Schouten J, Wit FW, Stolte IG, et al. Cross-sectional comparison of the prevalence of age-associated comorbidities and their risk factors between HIV-infected and uninfected individuals: the AGEhIV cohort study. Clin Infect Dis. 2014:59(12):1787-97. doi:10.1093/cid/ciu701.

6. Asboe D, Aitken C, Boffito M, et al. British HIV Association guidelines for the routine investigation and monitoring of adult HIV-1-infected individuals 2011. HIV Med. 2012;13(1):1-44. doi:10.1111/j.1468-1293.2011.00971.x.

7. British HIV Association Audit and Standards Sub-Committee. Routine monitoring and assessment of adults with HIV. http://bhiva.org/documents/
Conferences/Autumn2015/Presentations/151112/AoifeMolloy.pdf (accessed 21 Oct 2016).

8. Michael S, Gompels M, Sabin C, Curtis H, May MT. Benchmarked performance charts to improve the effectiveness of feedback of audit data in HIV care. BMC Health Services Research. 2017;17:506. https://doi.org/10. 1186/s12913-017-2426-6.

9. Ahn JY, Boettiger D, Law M, et al. Implementation and operational research: effects of CD4 monitoring frequency on clinical end points in clinically stable HIV-infected patients with viral suppression. J Acquir Immune Defic Syndr. 2015;69(3):e85-92. doi:10.1097/QAl.0000000000000634.

10. Sax PE, Islam $R$, Walensky RP, et al. Should resistance testing be performed for treatment-naive HIV-infected patients? A cost-effectiveness analysis. Clin Infect Dis. 2005:41(9):1316-23. doi:10.1086/496984.

11. Booth C. Transmitted resistance. In: Geretti AM, ed. Antiretroviral Resistance in Clinical Practice. London: Mediscript; 2006.

12. Helleberg M, May MT, Ingle SM, et al. Smoking and life expectancy among HIV-infected individuals on antiretroviral therapy in Europe and North America. AIDS. 2015;29(2):221-9. doi:10.1097/QAD.0000000000000540.

13. Althoff KN, Smit M, Reiss $P$, Justice $A C$. HIV and ageing: improving quantity and quality of life. Curr Opin HIV AIDS. 2016;11(5). http://journals.Iww.com/ co-hivandaids/Fulltext/2016/09000/HIV_and_ageing__improving_quantity_ and quality of.12.aspx.

14. Geretti AM, British HIV. Association guidelines for immunization of HIVinfected adults 2008. HIV Med. 2008;9(10):795-848. doi:10.1111/j.1468-1293. 2008.00637.x

\section{Submit your next manuscript to BioMed Central and we will help you at every step:}

- We accept pre-submission inquiries

- Our selector tool helps you to find the most relevant journal

- We provide round the clock customer support

- Convenient online submission

- Thorough peer review

- Inclusion in PubMed and all major indexing services

- Maximum visibility for your research

Submit your manuscript at www.biomedcentral.com/submit
Biomed Central 